\title{
Fluorescent Properties of Hymecromone and Fluorimetric Analysis of Hymecromone in Compound Dantong Capsule
}

\author{
Huanhuan Zhi, ${ }^{1,2}$ Jingdan Wang, ${ }^{2}$ Shujing Wang, ${ }^{2}$ and Yongju Wei ${ }^{1,2}$ \\ ${ }^{1}$ College of Life Science, Hebei Normal University, Shijiazhuang 050024, China \\ ${ }^{2}$ College of Chemistry and Material Science, Hebei Normal University, Shijiazhuang 050024, China \\ Correspondence should be addressed to Yongju Wei; weiyju@126.com
}

Received 5 September 2013; Accepted 21 October 2013

Academic Editor: Yizhuang Xu

Copyright (c) 2013 Huanhuan Zhi et al. This is an open access article distributed under the Creative Commons Attribution License, which permits unrestricted use, distribution, and reproduction in any medium, provided the original work is properly cited.

\begin{abstract}
Fluorescence spectra of hymecromone (4MU) aqueous solutions are investigated at different pHs. Two fluorescent species of $4 \mathrm{MU}$, neutral molecular form and anion form, are considered to be the main fluorescent forms. Quantum yields of the two forms are measured to be 0.74 at $\mathrm{pH} 5.98$ and 0.95 at $\mathrm{pH} 9.75$, respectively. The ionization constant of 7-hydroxyl proton of $4 \mathrm{MU}$ is determined to be $\mathrm{p} K_{a}=7.85 \pm 0.03$ by a $\mathrm{pH}$-fluorescence method. Addition of methanol into $4 \mathrm{MU}$ aqueous solution leads to a blue shift of maximum emission wavelength from $445 \mathrm{~nm}$ to $380 \mathrm{~nm}$, and a decrease in fluorescence intensity. 3D fluorescence spectra of Chinese patent drug Compound Dantong Capsule (CDC) and its four component herbal drugs are also investigated. Based on their fluorescent properties, a novel fluorimetric method is proposed for the selective determination of 4MU in CDC without preseparation. The new method is suitable for the routine quality evaluation of CDC.
\end{abstract}

\section{Introduction}

Coumarins, both natural and synthetic, are of interest because of their multiple biological and photodynamic activities. They are extensively used as medicine and analytical reagent [1-7]. Hymecromone (4-Methyl-7-hydroxycoumarin, 7-Hydroxy-4-methylcoumarin, 4-Methylumbelliferone, abbreviated as $4 \mathrm{MU}$ ) is a synthetic coumarin compound. It is claimed that $4 \mathrm{MU}$ and its derivatives or metal complexes possess diverse pharmacological properties, such as antiviral [8], antifungal and antioxidative [9], anticancer [10$13]$, anticoagulant, and spasmolytic activities [14, 15]. The fluorescence of $4 \mathrm{MU}$ had been observed early [16], a watersoluble polymeric fluorescent probe for measurement of near-neutral $\mathrm{pH}$ was synthesized based on the fluorescent properties of $4 \mathrm{MU}$ [17], and the effect of $\mathrm{pH}$ on fluorescence was restudied recently [18]; however, no comprehensive and detailed fluorescence spectra and quantum yield have been found in the literature.

Compound Dantong Capsule (CDC, also named Fufang Dantong Capsule) is a Chinese proprietary medicine [19]. It is effective in cure cholecystitis, cholangitis, intercurrent infection of gallbladder, and biliary tract concretion $[19,20]$.
The chemical composition of CDC includes 4MU (also named Dantong) and four kinds of Chinese herbal medicines, including Herba Isodonis Lophanthoidis (Xihuangcao), Herba Artemisiae Scopariae (Yinchen), Herba Andrographis (Chuanxinlian), and Rhei Radix et Rhizoma (Dahuang). The content of $4 \mathrm{MU}$ is one of the main quality indexes of CDC [21]. Earlier, the spectrophotometric method was applied for the determination of $4 \mathrm{MU}$ in CDC according to the absorbance of $4 \mathrm{MU}$ at $372 \mathrm{~nm}$ [19]. Later, since the interference of coexistent components, high performance liquid chromatographic methods (HPLC) were developed for the determination of $4 \mathrm{MU}$ in CDC [21-23]. So far, no fluorimetric method was reported for the determination of $4 \mathrm{MU}$ in $\mathrm{CDC}$ and other medicinal samples.

Fluorimetry has been recognized as one of the most useful analytical methods owing to its high sensitivity, good selectivity, simplicity, speediness, and low cost. It can be suitable for analysis of some complex samples such as traditional Chinese medicine containing a fluorescent component, especially in the case of a routine analysis. Three-dimensional fluorescence spectra (3D fluorescent fingerprint) have been used in our lab for qualitative identification of Chinese herbal medicines 


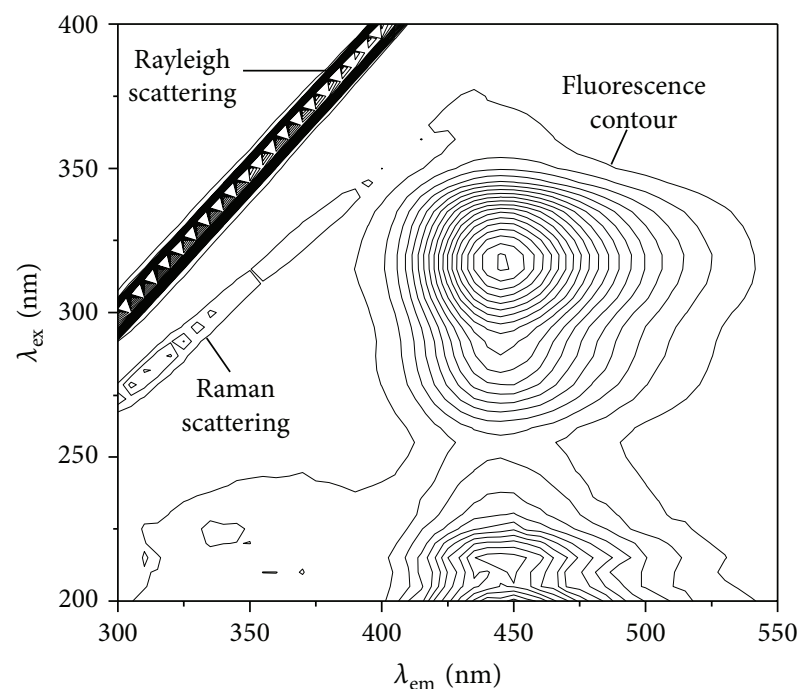

(a)

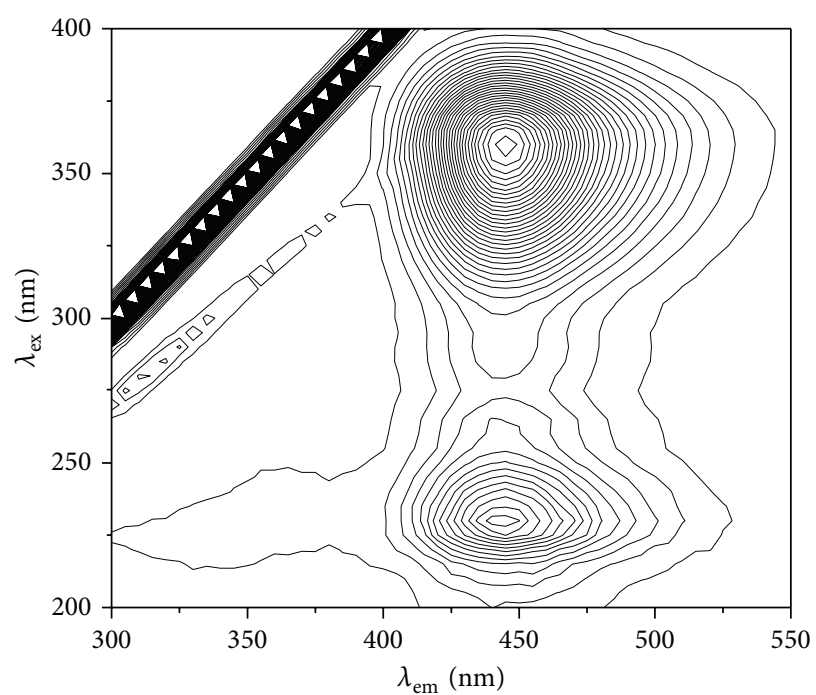

(b)

Figure 1: 3D fluorescence spectra of $4 \mathrm{MU}$ aqueous solution at different $\mathrm{pH}$. $c_{4 \mathrm{MU}}$ : $18.5 \mathrm{ng} \mathrm{mL}^{-1}$. (a) $\mathrm{pH} 5.98$; (b) $\mathrm{pH}$ 9.75. Contour interval: $R$.

[24]. Moreover, a number of fluorimetric methods have been developed for quantitative determination of active components in medicinal materials, such as paeonol in Cynanchi Paniculati Radix (Xuchangqing) [25], camptothecin in common Camptotheca fruit (Xishuguo) [26], and arctiin in Arctii Fructus (Niubangzi) [27]. In this study, the fluorescent properties of $4 \mathrm{MU}$ are investigated, and its quantum yield and 7-hydroxyl proton ionization constant are measured. The fluorescent properties of CDC and its four consisting Chinese herbal medicines are also studied. Based on the spectral differences between $4 \mathrm{MU}$ and other components in CDC, a fluorimetric method is proposed for the determination of $4 \mathrm{MU}$ in CDC sample without preseparation.

\section{Experimental}

2.1. Apparatus. Fluorescence measurements were performed on a Hitachi (Tokyo, Japan) F-7000 spectrofluorimeter equipped with a xenon lamp, $1 \mathrm{~cm}$ quartz cell, and a UV29 filter placed into the emission light path to remove secondary spectrum. The excitation and emission slits (band pass) $5 \mathrm{~nm} / 5 \mathrm{~nm}$ were used throughout the work. Absorption spectra were recorded using a Shimadzu (Kyoto, Japan) UV2501PC recording spectrophotometer with $1 \mathrm{~cm}$ quartz cell. An Orion (Beverly, USA) 868 pH/ISE meter was used for $\mathrm{pH}$ measurement.

2.2. Chemicals and Materials. Hymecromone (4MU, serial no. 100241-200503, a reagent for quantitative analysis, molecular weight: 176.17) and Chinese herbal medicine (comparison drug for qualitative identification) Xihuangcao (serial no. 121488-200501), Yinchen (serial no. 120950-200305), Chuanxinlian (serial no. 121082-200302), and Dahuang (serial no. 120984-200301) were purchased from the National Institute for the Control of Pharmaceuticals and Biological Products of China (Beijing, China). 4MU stock solution was prepared by dissolving $3.61 \mathrm{mg}$ reagent in $100 \mathrm{~mL}$ methanol (TEDIA, HPLC grade) and diluted to appropriate concentration with methanol as needed. Compound Dantong Capsule (CDC, product lot no. 100601) was produced by National Tsing Hua Pharmaceutical Co., Ltd. Hubei-day Saint, China. Quinine bisulphate (HPLC grade) was acquired from J\&K Scientific Ltd. (Beijing, China); its solution was prepared by dissolving $391.47 \mathrm{mg}$ reagent in $500 \mathrm{~mL} 0.05 \mathrm{~mol} \mathrm{~L}^{-1} \mathrm{H}_{2} \mathrm{SO}_{4}$ and diluted to appropriate concentration with $0.05 \mathrm{~mol} \mathrm{~L}^{-1}$ $\mathrm{H}_{2} \mathrm{SO}_{4}$ when it was used. Britton-Robinson buffer solution was a mixture of phosphoric acid, boric acid, and acetic acid (each $0.02 \mathrm{~mol} \mathrm{~L}^{-1}$ ) and adjusted to an appropriate $\mathrm{pH}$ by addition of $0.1 \mathrm{~mol} \mathrm{~L}^{-1} \mathrm{NaOH}$ solution. All the buffer chemicals were of analytical grade. The water used throughout the study was doubly-deionized and verified to be free from fluorescence.

2.3. General Procedure for Spectral Measurement. A series of $10 \mathrm{~mL}$ volumetric flasks was added appropriate amount of $4 \mathrm{MU}$ or CDC and buffer solutions. The mixtures were diluted to the mark with water and mixed well. Fluorescence or absorption spectra were measured at room temperature. Meanwhile, Raman scattering of water at excitation wavelength of $350 \mathrm{~nm}$ was measured (expressed as $R$, for calibration of 3D fluorescence spectra) [24].

2.4. Determination of Ionization Constant. A number of solutions containing the same concentration of weak acid $\mathrm{HB}$ and different $\mathrm{pH}$ were prepared. Fluorescence intensity $F$ and $\mathrm{pH}$ of each solution were measured. Then the ionization constant $\mathrm{p} K_{a}$ was calculated according to the following equation [28]:

$$
\mathrm{p} K_{a}=\mathrm{pH}-\lg \frac{F_{\mathrm{HB}}-F}{F-F_{\mathrm{B}}},
$$




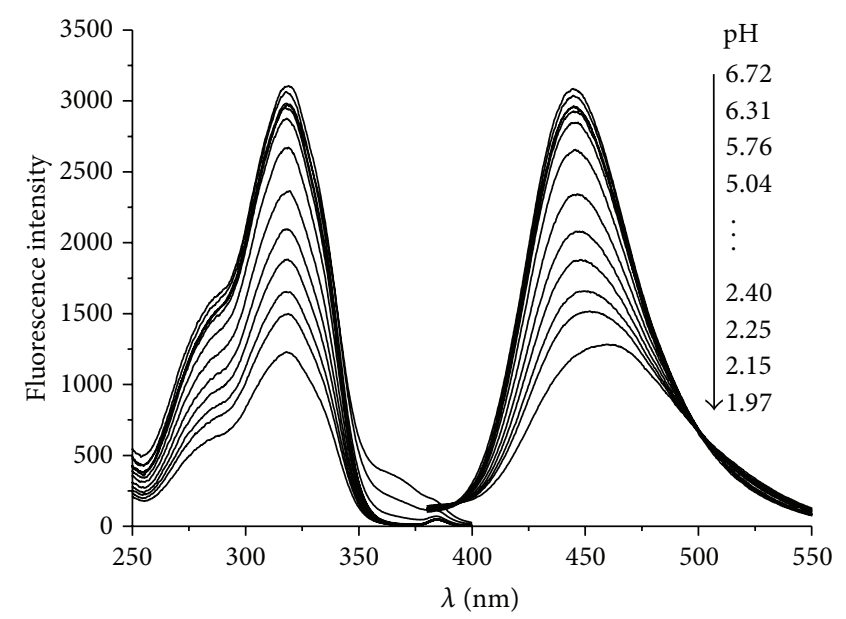

(a)

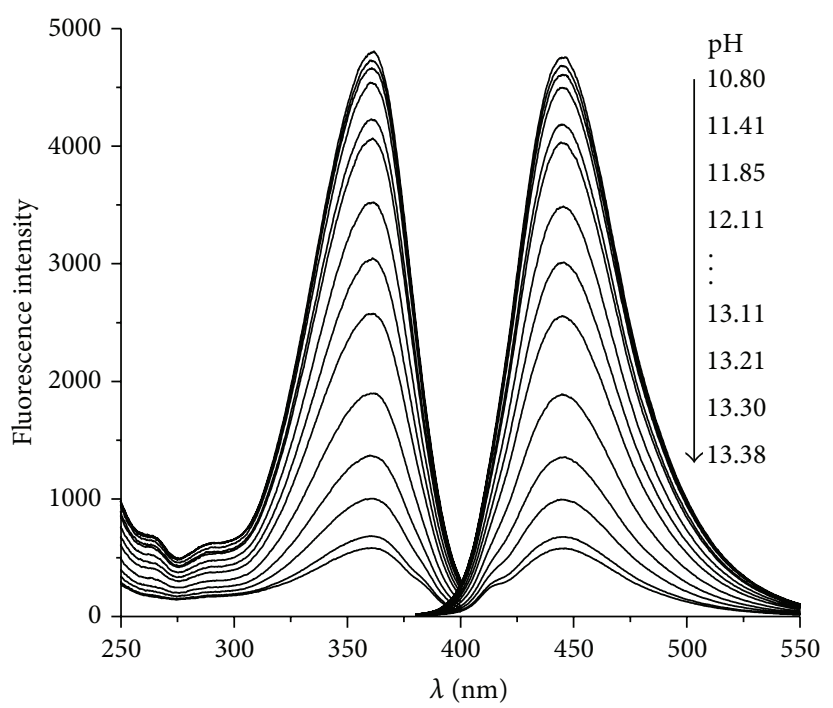

(c)

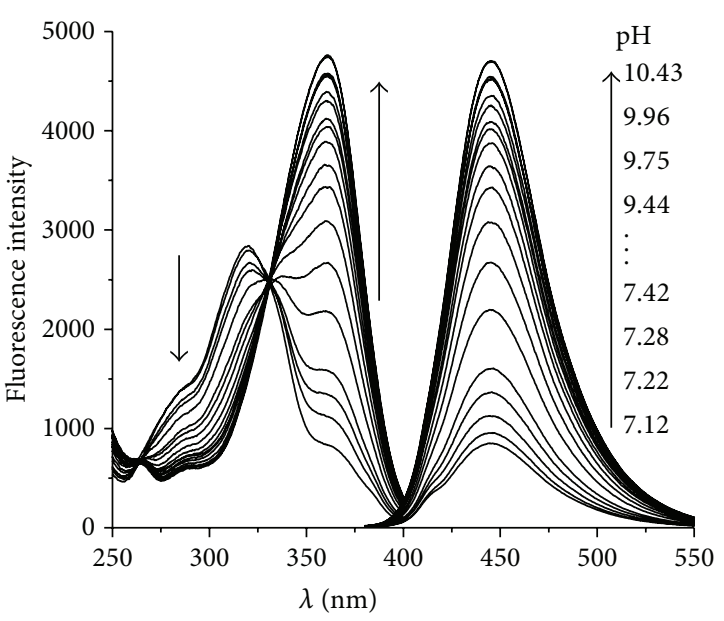

(b)

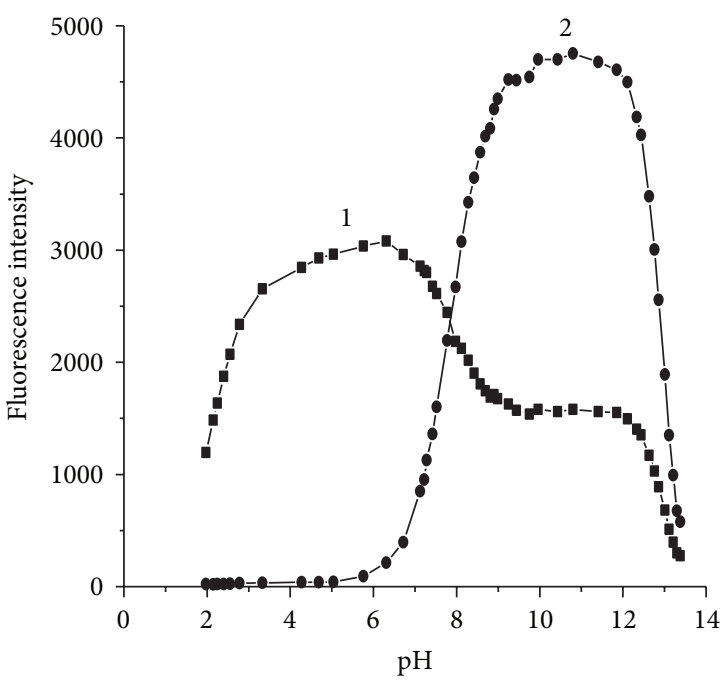

(d)

FIGURE 2: (a), (b) and (c) Fluorescence excitation and emission spectra of $4 \mathrm{MU}$ at different $\mathrm{pH}$ and (d) relationship between fluorescence intensity and pH. $c_{4 \mathrm{MU}}: 18.5 \mathrm{ng} \mathrm{mL}^{-1} ; \lambda_{\mathrm{ex}} / \lambda_{\mathrm{em}}$ : (a) and (d(1)):320 nm/445 nm, (b), (c) and (d(2)): $360 \mathrm{~nm} / 445 \mathrm{~nm}$.

where $F_{\mathrm{HB}}$ and $F_{\mathrm{B}}$ were the fluorescence intensity of $\mathrm{HB}$ and its conjugate base $\mathrm{B}$; they can be measured at sufficient acidic $\mathrm{pH}$ where all $\mathrm{HB}$ species exist in the neutral molecular form and at sufficient alkaline $\mathrm{pH}$ where all $\mathrm{HB}$ species exist in the anion form, respectively.

2.5. Measurement of Fluorescence Quantum Yield. Quinine bisulphate was used as a reference (quantum yield 0.55 at excitation wavelength of $313 \mathrm{~nm}$ ) in measuring quantum yield of $4 \mathrm{MU}$. For the measurement, quinine bisulphate and $4 \mathrm{MU}$ solutions were prepared in proper concentration so that the absorbance $(A)$ of the two solutions was similar and not larger than 0.05. Absorption and fluorescence spectra were recorded, and then quantum yields were calculated according to the following equation $[28,29]$ :

$$
Y_{u}=Y_{r} \cdot \frac{F_{u}}{F_{r}} \cdot \frac{A_{r}}{A_{u}}
$$

where $Y_{u}$ and $Y_{r}$ were the fluorescence quantum yield of unknown and the reference, $F_{u}$ and $F_{r}$ were the integral fluorescence intensity of unknown and reference solutions, and $A_{u}$ and $A_{r}$ were the absorbance of unknown and reference solutions at their excitation wavelengths, respectively.

2.6. Sample Preparation. Dissolve $9.21 \mathrm{mg}$ CDC powder in $100 \mathrm{~mL}$ methanol and dilute to $0.921 \mu \mathrm{g} \mathrm{mL}^{-1}$ with methanol as sample solution.

\section{Results and Discussion}

\subsection{Fluorescence Properties of $4 M U$}

3.1.1. 3D Fluorescence Spectra of 4MU. 3D (three-dimensional) fluorescence spectra of solvent blank and $4 \mathrm{MU}$ aqueous solutions at $\mathrm{pH} 5.98$ and at $\mathrm{pH} 9.75$ were measured as shown in Figure 1. The spectra indicated that the solvent used in 


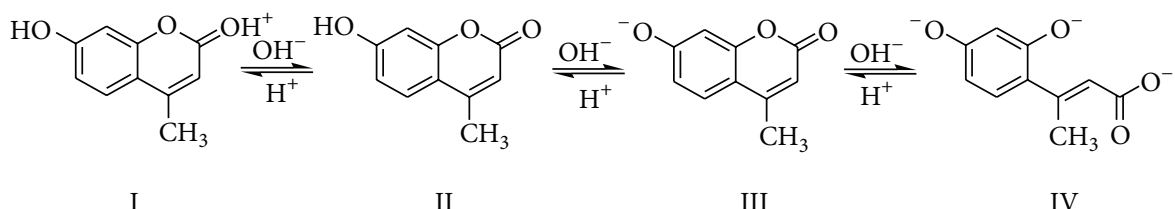

FIGURE 3: Proton ionization and hydrolysis process of $4 \mathrm{MU}$.

this study was basically no fluorescence. $4 \mathrm{MU}$ can produce strong fluorescence in near neutral condition with maximum excitation wavelength $\left(\lambda_{\text {ex }}\right)$ of $320 \mathrm{~nm}$ and maximum emission wavelength $\left(\lambda_{\mathrm{em}}\right)$ of $445 \mathrm{~nm}$, while in weak alkaline condition, the fluorescence intensity enhanced, $\lambda_{\text {ex }}$ red shifted from $320 \mathrm{~nm}$ to $360 \mathrm{~nm}$, but $\lambda_{\mathrm{em}}$ remained constant. Figure 1 revealed that $\mathrm{pH}$ has an important influence on fluorescence.

3.1.2. Effect of $p H$ on Fluorescence. As shown in Figure 2, effect of $\mathrm{pH}$ on fluorescence excitation and emission spectra of $4 \mathrm{MU}$ at various $\mathrm{pHs}$ were studied. In acidic conditions $(\mathrm{pH}$ 1.97-6.72), along with the decrease in $\mathrm{pH}$, fluorescence intensity declined, $\lambda_{\mathrm{ex}}$ centered at $320 \mathrm{~nm}$, and $\lambda_{\mathrm{em}}$ red shifted slightly from $445 \mathrm{~nm}$ to $455 \mathrm{~nm}$. In the range of $\mathrm{pH} 7.12-$ 10.43 , along with the increase in $\mathrm{pH}$, fluorescence emission at $445 \mathrm{~nm}$ enhanced, but the excitation band centered at $320 \mathrm{~nm}$ declined, meanwhile a new excitation band centered at $360 \mathrm{~nm}$ emerged and an iso-fluorescence point [30] formed at $330 \mathrm{~nm}$. In the range of $\mathrm{pH} 10.80-13.38$, along with the increase in $\mathrm{pH}$, fluorescence emission at $445 \mathrm{~nm}$ gradually quenched while $\lambda_{\mathrm{ex}}$ and $\lambda_{\mathrm{em}}$ remained constant.

The relationship between $\mathrm{pH}$ and fluorescence intensity was summarized in Figure 2(d).

The variation of fluorescence spectra implies that the solution equilibrium or molecular structure of $4 \mathrm{MU}$ changed along with the change in $\mathrm{pH}$. According to Figure 2, we suppose that the proton dissociation and hydrolysis process of $4 \mathrm{MU}$ is as shown in Figure 3.

The structure of $4 \mathrm{MU}$ contains a benzene ring (linked with 7-OH) and a lactonic ring (include lactone bond and $2-\mathrm{C}=\mathrm{O}$ ). In strong acid conditions, the 2-carbonyl oxygen protonated $[28,30]$ to form a cationic species (type I), leading to a decrease in fluorescence intensity and a red shift in emission wavelength. In near neutral conditions ( $\mathrm{pH}$ 4.0-7.0), 4MU exist mainly as molecular form (type II) which have strong fluorescence with $\lambda_{\text {ex }}$ of $320 \mathrm{~nm}$ and $\lambda_{\text {em }}$ of $445 \mathrm{~nm}$. In weak alkaline conditions ( $\mathrm{pH}$ 9.0-12.0), 7-hydroxyl proton dissociated and 4MU exist mainly as anion form (type III) which have stronger fluorescence with $\lambda_{\mathrm{ex}}$ of $360 \mathrm{~nm}$ and $\lambda_{\text {em }}$ of $445 \mathrm{~nm}$. In strong alkaline conditions ( $\mathrm{pH}>12.0$ ), the hydrolysis of lactone bond take place $[28,31]$, leading to a fluorescence quenching.

3.1.3. Ionization Constant of 7-Hydroxyl Proton. With the above discussion, ionization constant of 7-OH proton can be determined in suitable conditions. Using the $\mathrm{pH}$-fluorescence method described in Section 2.4, the ionization constant of 7-hydroxyl proton was determined to be $\mathrm{p} K_{a}=7.85 \pm 0.03$ (Table 1).
TABLE 1: Determination of ionization constant of $4 \mathrm{MU}$.

\begin{tabular}{ccccc}
\hline $\mathrm{pH}$ & $F$ & $\lg \left[\left(F_{\mathrm{HB}}-F\right) /\left(F-F_{\mathrm{B}}\right)\right]^{*}$ & $\mathrm{p} K_{a}$ & Average $\mathrm{p} K_{a}$ \\
\hline 7.52 & 1601 & -0.30 & 7.82 & \\
7.77 & 2195 & -0.07 & 7.84 & \\
7.98 & 2672 & 0.11 & 7.87 & $7.85 \pm 0.03$ \\
8.12 & 3074 & 0.26 & 7.86 & \\
8.28 & 3427 & 0.41 & 7.87 & \\
\hline
\end{tabular}

${ }^{*} F_{\mathrm{HB}}=21.18, F_{\mathrm{B}}=4753$.

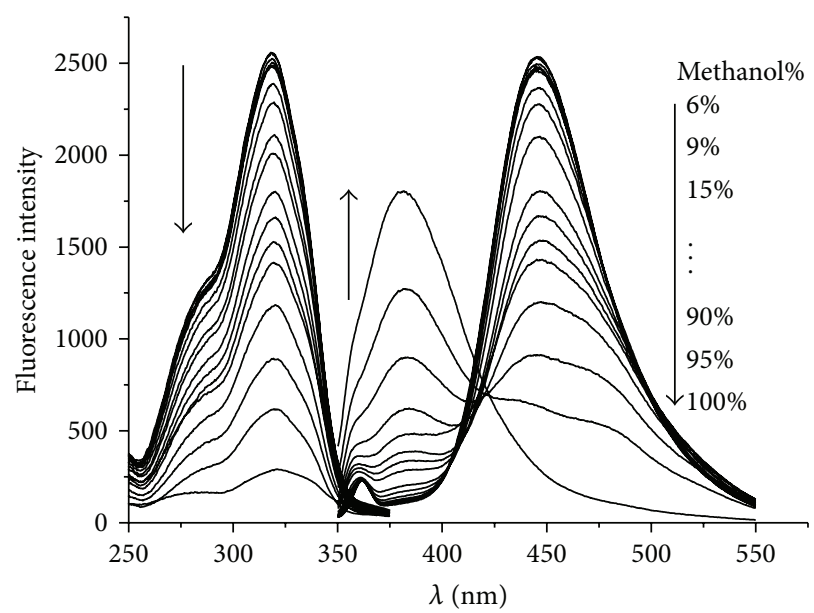

FIGURE 4: Fluorescence spectra of $4 \mathrm{MU}$ in aqueous solutions containing different amounts of methanol. $c_{4 \mathrm{MU}}: 18.5 \mathrm{ng} \mathrm{mL}^{-1} ; \mathrm{pH}$ : $5.98 ; \lambda_{\mathrm{ex}} / \lambda_{\mathrm{em}}: 320 \mathrm{~nm} / 445 \mathrm{~nm}$.

In addition, according to the relationship between fluorescence intensity and $\mathrm{pH}$, we can graphically estimate the ionization constant. In Figure 2(d), the abscissa of the intersection of curve (a) and curve (b) is about 7.85, which is just the $\mathrm{p} K_{a}$.

The ionization constant determined above is close to the ionization constant of umbelliferone; $\mathrm{p} K_{a}=7.61 \pm 0.03$ [28]. The little difference between the two constants reflects the impact of 4-methyl on the acidity of 7-hydroxyl proton.

3.1.4. Effect of Solvent on Fluorescence. Solvent polarity usually has a profound effect on the emission spectral properties of fluorophores and further influences the sensitivity of a fluorimetric method. Figure 4 shows the fluorescence spectra of $4 \mathrm{MU}$ in aqueous solutions containing different amounts of methanol. Along with the increase percentage of methanol, fluorescence emission band centered at $445 \mathrm{~nm}$ gradually 


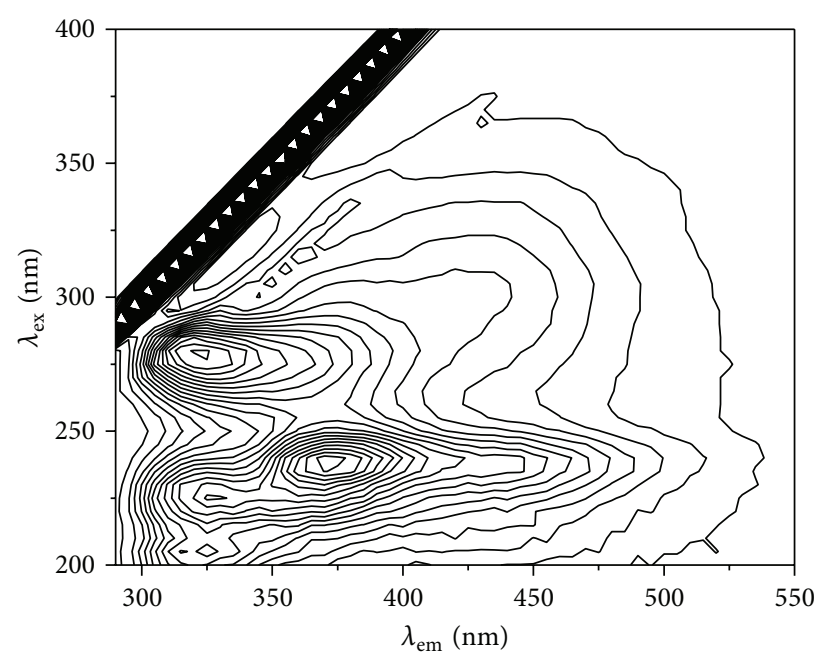

(a)

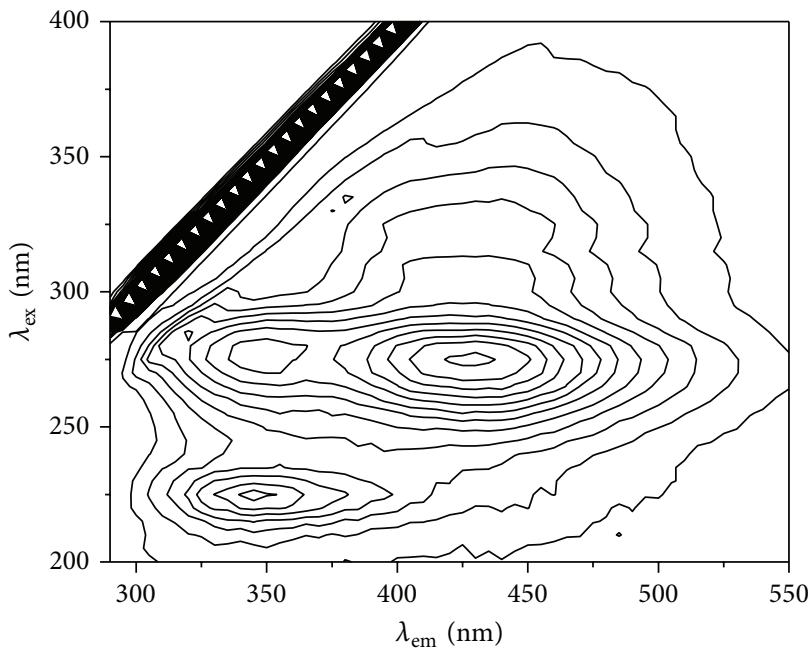

(c)

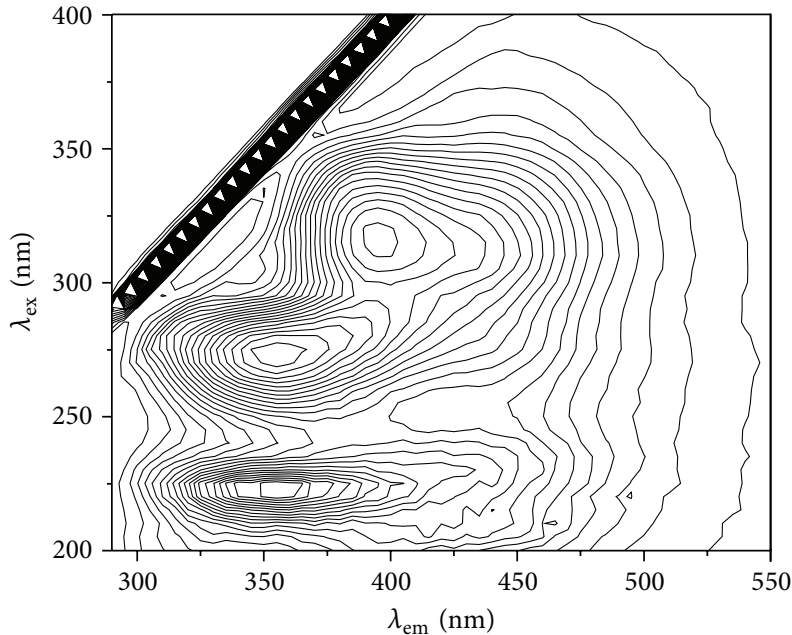

(b)

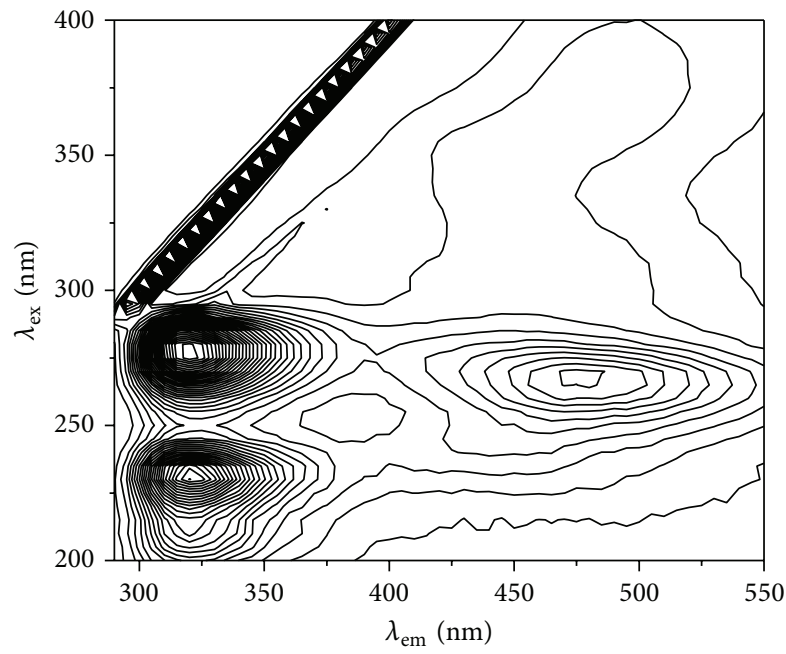

(d)

FIGURE 5: 3D fluorescence spectra of four kinds of Chinese herbal medicines in CDC. (a) Xihuangcao, $c: 400 \mu \mathrm{g} \mathrm{mL} L^{-1}$; (b) Yinchen, $c$ : $400 \mu \mathrm{g} \mathrm{mL}{ }^{-1}$; (c) Chuanxinlian, $c: 400 \mu \mathrm{g} \mathrm{mL}^{-1}$; (d) Dahuang, $c: 100 \mu \mathrm{g} \mathrm{mL}{ }^{-1}$. Contour interval: $R$.

declined, while a new emission band centered at $380 \mathrm{~nm}$ emerged. When the percentage of methanol reached $100 \%$, the $\lambda_{\mathrm{em}}$ blue shifted to $380 \mathrm{~nm}$; however, the fluorescence intensity was less than that in aqueous solutions. Therefore, we choose water as the appropriate solvent in establishing a fluorimetric method in the following study, and control the methanol percentage in aqueous solution no more than $20 \%$ to obtain sensitive and stable fluorescence.

3.1.5. Measurement of Fluorescence Quantum Yield of $4 M U$. Using the method described in Section 2.5, quantum yields of $4 \mathrm{MU}$ in near neutral $(\mathrm{pH} 5.98)$ and weak alkaline $(\mathrm{pH}$ 9.75) conditions were measured, as shown in Table 2. In near neutral solution, $4 \mathrm{MU}$ exist as molecular form; its quantum yield at excitation wavelength $320 \mathrm{~nm}$ was measured to be 0.74 . In weak alkaline solution, $4 \mathrm{MU}$ exist as anion form; its quantum yield at excitation wavelength $360 \mathrm{~nm}$ was measured to be 0.95 . These results indicate that $4 \mathrm{MU}$ is an excellent fluorophore. A fluorimetric method for determination of $4 \mathrm{MU}$ in Chinese patent drug should be very sensitive either in near neutral or in weak alkaline conditions.

\subsection{Determination of $4 M U$ in Compound Dantong Capsule}

3.2.1. 3D Fluorescence Spectra of Four Kinds of Chinese Herbal Medicines in $C D C$. The key point for developing a fluorimetric method is to understand the fluorescent properties of the analyte and other components in the sample, then find a way to avoid interference of the coexistent components on the fluorescence of the analyte. For these reason, 3D fluorescence spectra of four kinds of Chinese herbal materials in CDC were measured and shown in Figure 5.

From Figure 5 we showed that all the four kinds of Chinese herbal medicines contain fluorescent components. The interference of these components on the fluorescence of $4 \mathrm{MU}$ seems to be a serious problem. However, we noted 


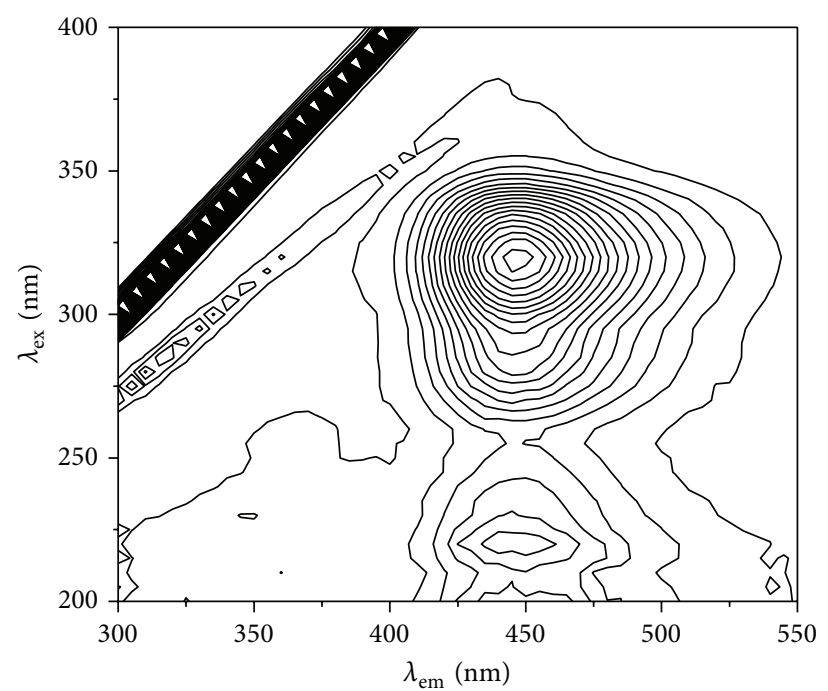

(a)

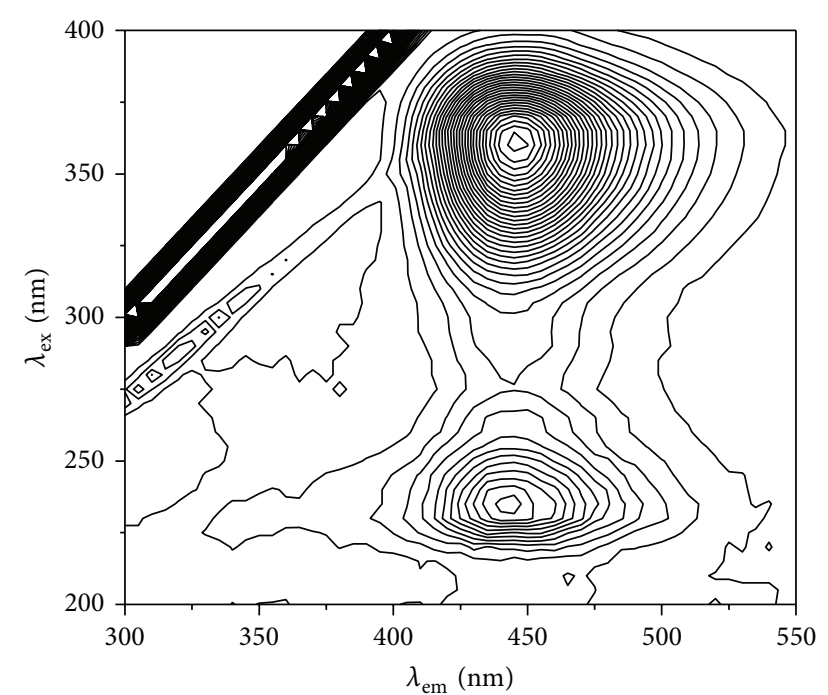

(b)

Figure 6: 3D fluorescence spectra of CDC solution at different $\mathrm{pH} . c_{\mathrm{CDC}}: 55.3 \mathrm{ng} \mathrm{mL} \mathrm{L}^{-1}$. (a) $\mathrm{pH} 5.85$; (b) $\mathrm{pH}$ 9.22. Contour interval: $R$.

TABLE 2: Measurement of fluorescence quantum yield of $4 \mathrm{MU}$ in different condition.

\begin{tabular}{lcccccccc}
\hline \multirow{2}{*}{$\lambda_{\text {ex }} / \mathrm{nm}$} & \multicolumn{3}{c}{ Quinine bisulphate } & \multicolumn{3}{c}{ 4MU (pH 5.98) } & \multicolumn{2}{c}{4 4MU (pH 9.75) } \\
\hline 313 & $A$ & $F^{*}$ & $Y$ & $A$ & $F^{*}$ & $Y$ & $F^{*}$ \\
320 & 0.0365 & 105566 & 0.55 & 0.0440 & 178748 & 0.77 & & \\
350 & 0.0391 & 104878 & 0.51 & 0.0488 & 190213 & 0.74 & 0.0440 & 220646 \\
360 & 0.0411 & 123457 & 0.57 & & & & 0.95 \\
\hline
\end{tabular}

$F^{*}$ wavelength range of integral fluorescence intensity: $380 \mathrm{~nm} \sim 600 \mathrm{~nm}$.

that the concentrations of these herbal medicines in Figure 5 $\left(400 \mu \mathrm{g} \mathrm{mL}^{-1} \sim 100 \mu \mathrm{g} \mathrm{mL}^{-1}\right)$ were much higher than the concentration of $4 \mathrm{MU}$ in Figure $1\left(18.5 \mathrm{ng} \mathrm{mL}^{-1}\right)$. So, whether these herbal medicines in CDC interfere with the fluorescence of $4 \mathrm{MU}$ should be considered further from the view point of concentration.

3.2.2. 3D Fluorescence Spectra of CDC. Figure 6 shows the 3D fluorescence spectra of CDC dilute solutions at $\mathrm{pH} 5.85$ and at $\mathrm{pH}$ 9.22. Comparing Figure 6 to Figure 1, we figured out that the two pictures are basically the same. The fluorescence peaks presented in Figure 5 are not emerged in Figure 6. This result indicated that the fluorescence of those four herbal medicines in CDC is too weak to be seen, either because of their low concentration or due to their poor luminous ability. So, we can conclude that the coexistent components in CDC do not interfere with the fluorescence of $4 \mathrm{MU}$. The content of $4 \mathrm{MU}$ in CDC can be determined simply by a fluorimetric method.

From Figures 6 and 2, we also know that the fluorimetric method may be performed at near neutral or at weak alkaline $\mathrm{pH}$. For simplicity, we prefer the method to be performed at near neutral $\mathrm{pH}$ so that the fluorescence intensity of CDC water solution can be measured directly without using a buffer solution.
3.2.3. Determination of $4 M U$ in CDC by a Standard Curve Method. A series of standard aqueous solutions containing different amounts of $4 \mathrm{MU}$, from 1.55 to $31.0 \mathrm{ng} \mathrm{mL}^{-1}$, were prepared. Fluorescence spectra, as shown in Figure $7(a)$, and fluorescence intensity at measuring wavelength of $\lambda_{\mathrm{ex}} / \lambda_{\mathrm{em}}=$ $320 \mathrm{~nm} / 445 \mathrm{~nm}$ of each solution were measured. A linear calibration curve of fluorescence intensity against concentration was plotted, as shown in Figure 7(b). The regression equation obtained was $I_{F}=5.5+104.1 c\left(\mathrm{ng} \mathrm{mL}^{-1}\right)$, with correlation coefficient $R=0.9998(n=13)$.

A number of solutions containing different amounts of CDC were prepared and their fluorescence intensities at wavelength of $\lambda_{\mathrm{ex}} / \lambda_{\mathrm{em}}=320 \mathrm{~nm} / 445 \mathrm{~nm}$ were recorded. Using the calibration curve, the $4 \mathrm{MU}$ content in CDC sample was determined to be $33.35 \pm 0.10 \%$, as shown in Table 3 .

3.2.4. Determination of $4 M U$ in CDC by a Standard Addition Method. As shown in Figure 8, a standard addition method was employed to verify the result obtained above. The regression equation obtained was $I_{F}=636.9+105.3 c\left(\mathrm{ng} \mathrm{mL}{ }^{-1}\right)$, with correlation coefficient $R=0.9998(n=5)$. The content of $4 \mathrm{MU}$ in CDC sample was calculated to be $33.3 \%$, which was coincident with the result obtained by standard curve method. 


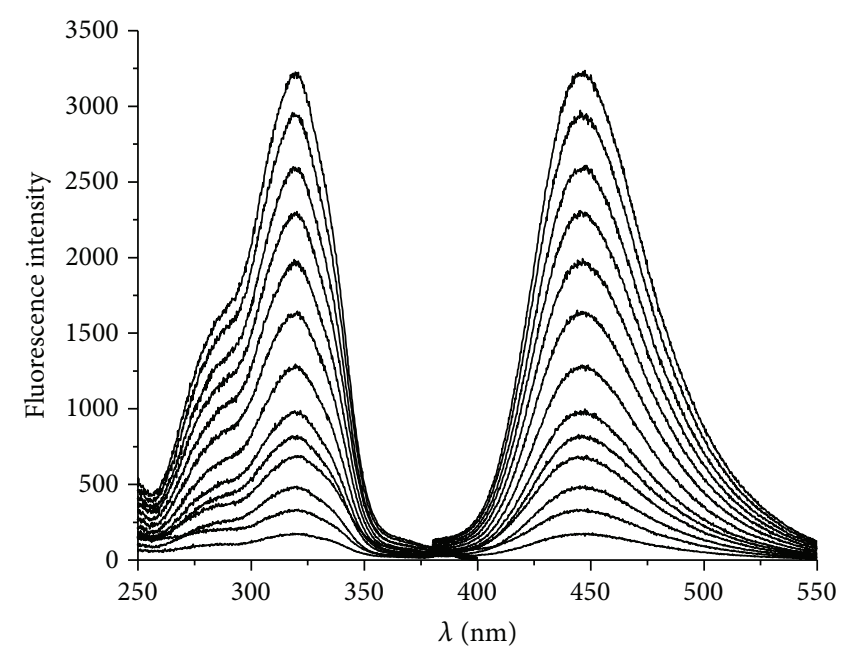

(a)

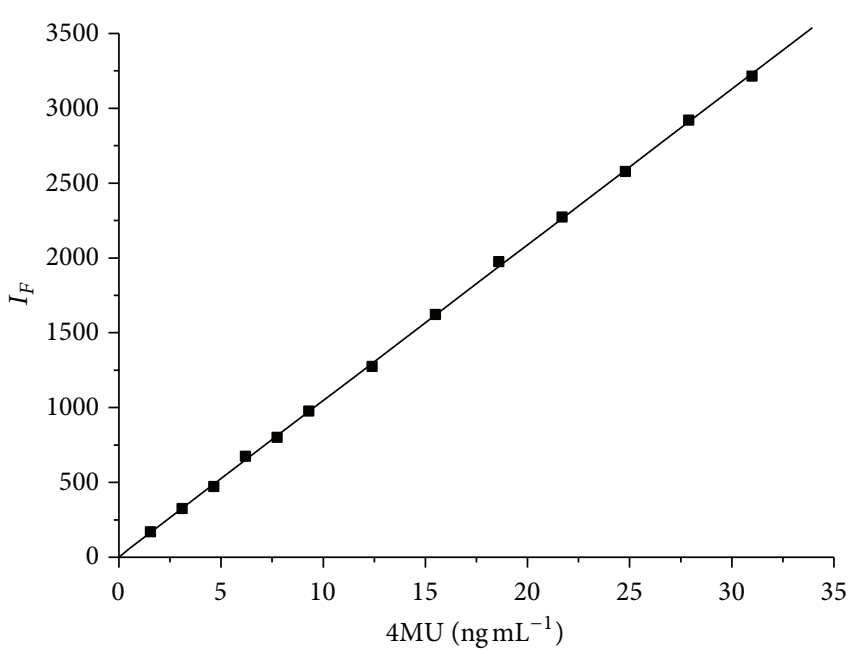

(b)

FIGURE 7: (a) Fluorescence spectra of 4MU in different concentrations; (b) plot of fluorescence intensity versus concentration. pH: 5.98; $\lambda_{\mathrm{ex}} / \lambda_{\mathrm{em}}: 320 \mathrm{~nm} / 445 \mathrm{~nm}$.

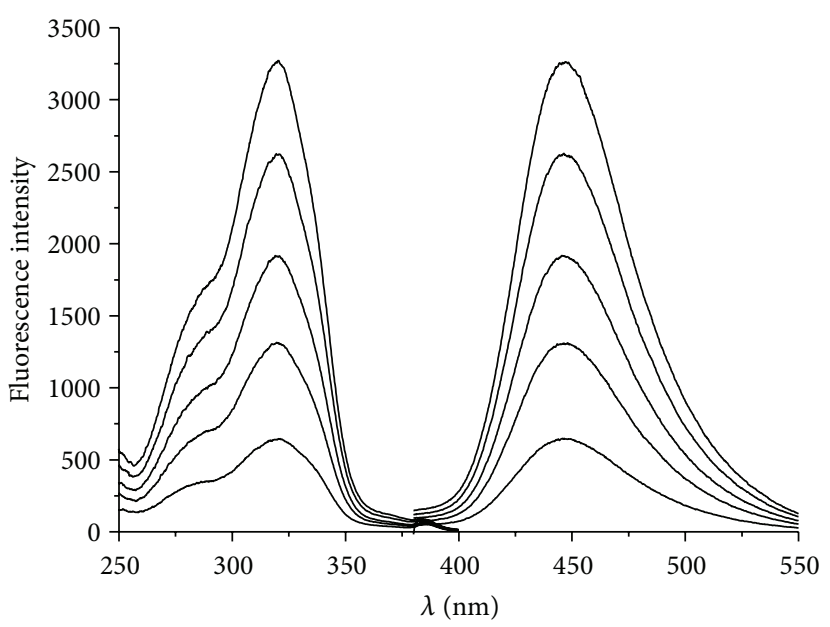

(a)

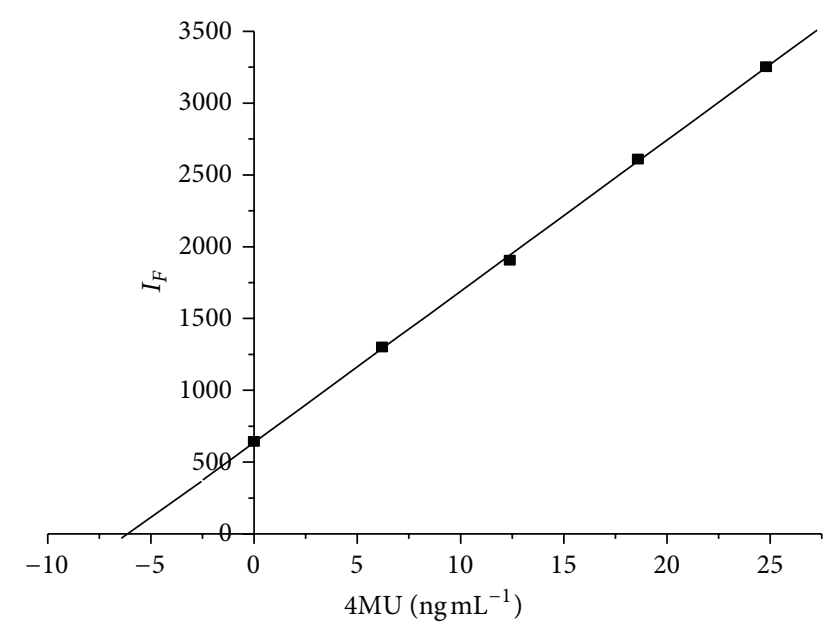

(b)

FIGURE 8: (a) Fluorescence spectra and (b) plot of fluorescence intensity versus concentration of a standard addition method for the determination of $4 \mathrm{MU}$ in CDC sample. $\mathrm{pH}: 5.98 ; \lambda_{\mathrm{ex}} / \lambda_{\mathrm{em}}: 320 \mathrm{~nm} / 445 \mathrm{~nm}$.

TABLE 3: Determination of 4MU in CDC by standard curve method.

\begin{tabular}{lccccc}
\hline No. & 1 & 2 & 3 & 4 & 5 \\
\hline CDC added $(\mathrm{V} / \mathrm{mL})$ & 0.20 & 0.40 & 0.60 & 0.80 & 1.00 \\
Fluorescence intensity $\left(I_{F}\right)$ & 643.5 & 1285 & 1924 & 2561 & 3217 \\
4MU determined $\left(\mathrm{ng} \mathrm{mL}^{-1}\right)$ & 6.13 & 12.3 & 18.4 & 24.5 & 30.8 \\
4MU content in CDC (\%) & 33.3 & 33.4 & 33.3 & 33.3 & 33.5 \\
\hline Average content $(\%)$ & \multicolumn{6}{c}{$33.4 \pm 0.1$}
\end{tabular}

According to the CDC drug label, the content of $4 \mathrm{MU}$ in CDC is $100 \mathrm{mg}$ per grain $(0.3 \mathrm{~g})$. It is evident that the analytical result of this method agrees with the drug label.

\section{Conclusion}

Hymecromone (4MU) is an excellent fluorophore. Acidity and solvent have important influence on its fluorescence. In near neutral aqueous solutions, $4 \mathrm{MU}$ exist mainly as neutral molecular form which can be produced strong fluorescence at $445 \mathrm{~nm}$. In weak alkaline solutions, $4 \mathrm{MU}$ exist mainly as anion form which can be produced stronger fluorescence at $445 \mathrm{~nm}$. In methanol solution, the fluorescence peak at $445 \mathrm{~nm}$ blue shifted to $380 \mathrm{~nm}$ and the fluorescence intensity declined in a certain extent. There is a good linear relationship between fluorescent intensity and 4MU concentration. The 3D fluorescence spectra of Chinese patent drug CDC dilute solutions are very similar to the $3 \mathrm{D}$ fluorescence spectra of $4 \mathrm{MU}$, indicating that the main fluorescent component in 
CDC is $4 \mathrm{MU}$. The coexistent components in CDC do not interfere with the fluorescence of $4 \mathrm{MU}$ because of their low concentration or poor luminous ability. So, the content of $4 \mathrm{MU}$ in CDC can be determined simply by fluorimetric method without preseparation.

\author{
Abbreviations \\ CDC: Compound Dantong Capsule (Fufang \\ Dantong Capsule) \\ $\lambda_{\mathrm{ex}}:$ Maximum excitation wavelength \\ $\lambda_{\mathrm{em}}$ : Maximum emission wavelength.
}

\section{Conflict of Interests}

The authors declare that they have no conflict of interests.

\section{Acknowledgment}

This work was supported by the National Natural Science Foundation of China (nos. 20675025, 20975029, and 81173496).

\section{References}

[1] Z. Zhou, N. Li, and A. Tong, "A new coumarin-based fluorescence turn-on chemodosimeter for $\mathrm{Cu}^{2+}$ in water," Analytica Chimica Acta, vol. 702, no. 1, pp. 81-86, 2011.

[2] L. Y. Wang, H. H. Li, and D. R. Cao, "A new photoresponsive coumarin-derived Schiff base: chemosensor selectively for $\mathrm{Al}^{3+}$ and $\mathrm{Fe}^{3+}$ and fluorescence "turn-on" under room light," Sensors and Actuators B, vol. 181, pp. 749-755, 2013.

[3] K. Li, N. Li, X. Chen, and A. Tong, "A ratiometric fluorescent chemodosimeter for $\mathrm{Cu}(\mathrm{II})$ in water with high selectivity and sensitivity," Analytica Chimica Acta, vol. 712, pp. 115-119, 2012.

[4] H. X. Xu, X. Q. Wang, C. L. Zhang, Y. P. Wu, and Z. P. Liu, "Coumarin-hydrazone based high selective fluorescence sensor for copper(II) detection in aqueous solution," Inorganic Chemistry Communications, vol. 34, pp. 8-11, 2013.

[5] X. B. Huang, Y. Dong, Q. W. Huang, and Y. X. Cheng, "Hydrogen bond induced fluorescence recovery of coumarinbased sensor system," Tetrahedron Letters, vol. 54, pp. 38223825, 2013.

[6] N. Chattopadhyay, A. Mallick, and S. Sengupta, "Photophysical studies of 7-hydroxy-4-methyl-8- ( $4^{\prime}$-methylpiperazin-1' -yl) methylcoumarin: a new fluorescent chemosensor for zinc and nickel ions in water," Journal of Photochemistry and Photobiology A, vol. 177, no. 1, pp. 55-60, 2006.

[7] P. Mladěnka, K. Macáková, L. Zatloukalová et al., "In vitro interactions of coumarins with iron," Biochimie, vol. 92, no. 9, pp. 1108-1114, 2010.

[8] M. Mazzei, E. Nieddu, M. Miele et al., "Activity of Mannich bases of 7-hydroxycoumarin against Flaviviridae," Bioorganic and Medicinal Chemistry, vol. 16, no. 5, pp. 2591-2605, 2008.

[9] B. Sarkanj, M. Molnar, M. Cacic, and L. Gille, "4-Methyl-7hydroxycoumarin antifungal and antioxidant activity enhancement by substitution with thiosemicarbazide and thiazolidinone moieties," Food Chemistry, vol. 139, pp. 488-495, 2013.

[10] H. Morohashi, A. Kon, M. Nakai et al., "Study of hyaluronan synthase inhibitor, 4-methylumbelliferone derivatives on human pancreatic cancer cell (KP1-NL)," Biochemical and Biophysical Research Communications, vol. 345, no. 4, pp. 14541459, 2006.

[11] S. S. Bhattacharyya, S. Paul, S. K. Mandal, A. Banerjee, N. Boujedaini, and A. R. Khuda-Bukhsh, "A synthetic coumarin (4-Methyl-7 hydroxy coumarin) has anti-cancer potentials against DMBA-induced skin cancer in mice," European Journal of Pharmacology, vol. 614, no. 1-3, pp. 128-136, 2009.

[12] K. Harada, H. Kubo, Y. Tomigahara et al., "Coumarins as novel $17 \beta$-hydroxysteroid dehydrogenase type 3 inhibitors for potential treatment of prostate cancer," Bioorganic and Medicinal Chemistry Letters, vol. 20, no. 1, pp. 272-275, 2010.

[13] A. Kultti, S. Pasonen-Seppänen, M. Jauhiainen et al., “4Methylumbelliferone inhibits hyaluronan synthesis by depletion of cellular UDP-glucuronic acid and downregulation of hyaluronan synthase 2 and 3," Experimental Cell Research, vol. 315, no. 11, pp. 1914-1923, 2009.

[14] I. P. Kostova, I. I. Manolov, I. N. Nicolova, and N. D. Danchev, "New metal complexes of 4-methyl-7-hydroxycoumarin sodium salt and their pharmacological activity," Il Farmaco, vol. 56, no. 9, pp. 707-713, 2001.

[15] I. Kostova, I. Manolov, I. Nicolova, S. Konstantinov, and M. Karaivanova, "New lanthanide complexes of 4-methyl-7hydroxycoumarin and their pharmacological activity," European Journal of Medicinal Chemistry, vol. 36, no. 4, pp. 339-347, 2001.

[16] L. D. Li and G. Z. Jin, "Luminescence properties of 4-Methyl7-hydroxy-coumarin," Chinese Journal of Analytical Chemistry, vol. 22, no. 5, pp. 440-444, 1994.

[17] S. Dong, H. Ma, X. Li, M. Sun, and X. Duan, "Synthesis of a new water-soluble polymeric probe and its fluorescent properties for ratiometric measurement of near-neutral pH," Analytical Letters, vol. 37, no. 14, pp. 2937-2948, 2004.

[18] I. Jones, J. Hamilton, R. Srivastava, and P. Galloway, "Effect of neutral and acid $\mathrm{pH}$ on the fluorescence of 4-methylumbelliferone and the implications for dry blood spot assays," Molecular Genetics and Metabolism, vol. 108, no. 2, article S51, 2013.

[19] Pharmacopoeia Committee of Chinese Ministry of Health, Traditional Chinese Medicine Drugs Preparation, vol. 5, 1991.

[20] A. Abate, V. Dimartino, P. Spina et al., "Hymecromone in the treatment of motor disorders of the bile ducts: a multicenter, double-blind, placebo-controlled clinical study," Drugs under Experimental and Clinical Research, vol. 27, no. 5-6, pp. 223-231, 2001.

[21] L. Zhang, B. Y. Li, and A. L. Yang, "Studies on the quality standard for Compound Dantong Capsule," Tianjin Pharmacy, vol. 16, no. 5, pp. 15-17, 2004.

[22] L. Zhang and J. Zhu, "Determination of the content and dissolution of hymecromone in Compound Dantong Capsules by HPLC," Traditional Chinese Drug Research \& Clinical Pharmacology, vol. 17, no. 3, pp. 222-224, 2006.

[23] L. R. Zheng, D. Liang, and Y. Chen, "Determination of 7hydroxy-4-methylcoumarin in Compound Dantong Capsule by HPLC," Pharmaceutical and Clinical Research, vol. 16, no. 4, pp. 333-334, 2008.

[24] Y. J. Wei, 3D Fluorescent Fingerprint of Chinese Herbal Medicine, Science Press, Beijing, China, 2012.

[25] F. Yang, C. G. Liu, and Y. J. Wei, "Fluorimetric analysis of paeonol in Chinese herbal medicine Cynanchi Paniculati Radix by aluminum ion-sensitized fluorescence," Acta Pharmaceutica Sinica B, vol. 2, no. 3, pp. 294-299, 2012. 
[26] L. P. Wang and Y. J. Wei, "Fluorimetric analysis of camptothecin in Chinese herbal medicine common Camptotheca fruit," Acta Pharmaceutica Sinica, vol. 47, no. 10, pp. 1370-1374, 2012.

[27] S. J. Wang, Q. Zhang, L. P. Wang, and Y. J. Wei, "Fluorescent properties of arctiin and arctigenin and fluorimetric analysis of arctiin in traditional Chinese medicine Arctii Fructus," Journal of Instrumental Analysis, vol. 33, 2014.

[28] J. Zhang, C. Liu, and Y. Wei, "Fluorescence quantum yield and ionization constant of umbelliferone," Huaxue Tongbao, vol. 74, no. 10, pp. 957-960, 2011.

[29] J. G. Xu and Z. B. Wang, Fluorimetry, Science Press, Beijing, China, 3rd edition, 2007.

[30] C. G. Liu, Y. Z. Xu, Y. J. Wei et al., "Fluorescence spectra and protonation of ofloxacin," Spectroscopy and Spectral Analysis, vol. 25, no. 4, pp. 584-587, 2005.

[31] L. Y. Kong, Coumarin Chemistry, Chemical Industry Press, Beijing, China, 1st edition, 2008. 

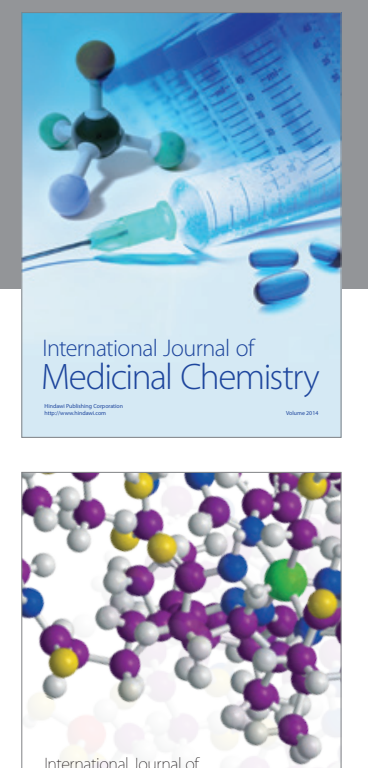

\section{Carbohydrate} Chemistry

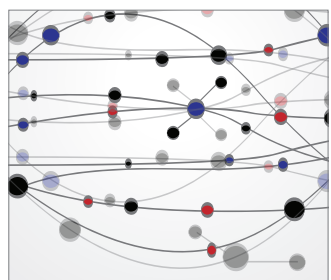

The Scientific World Journal
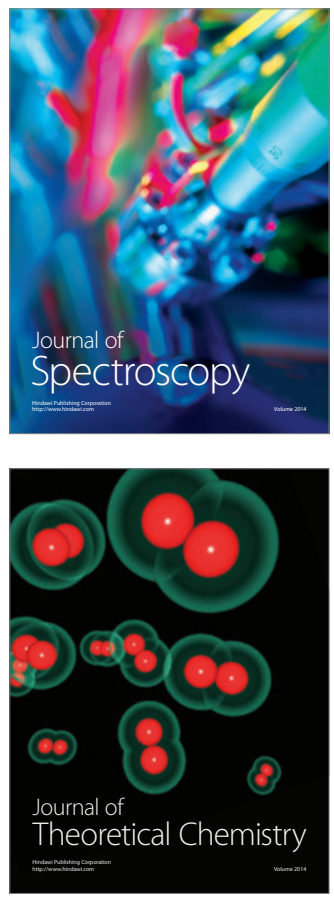
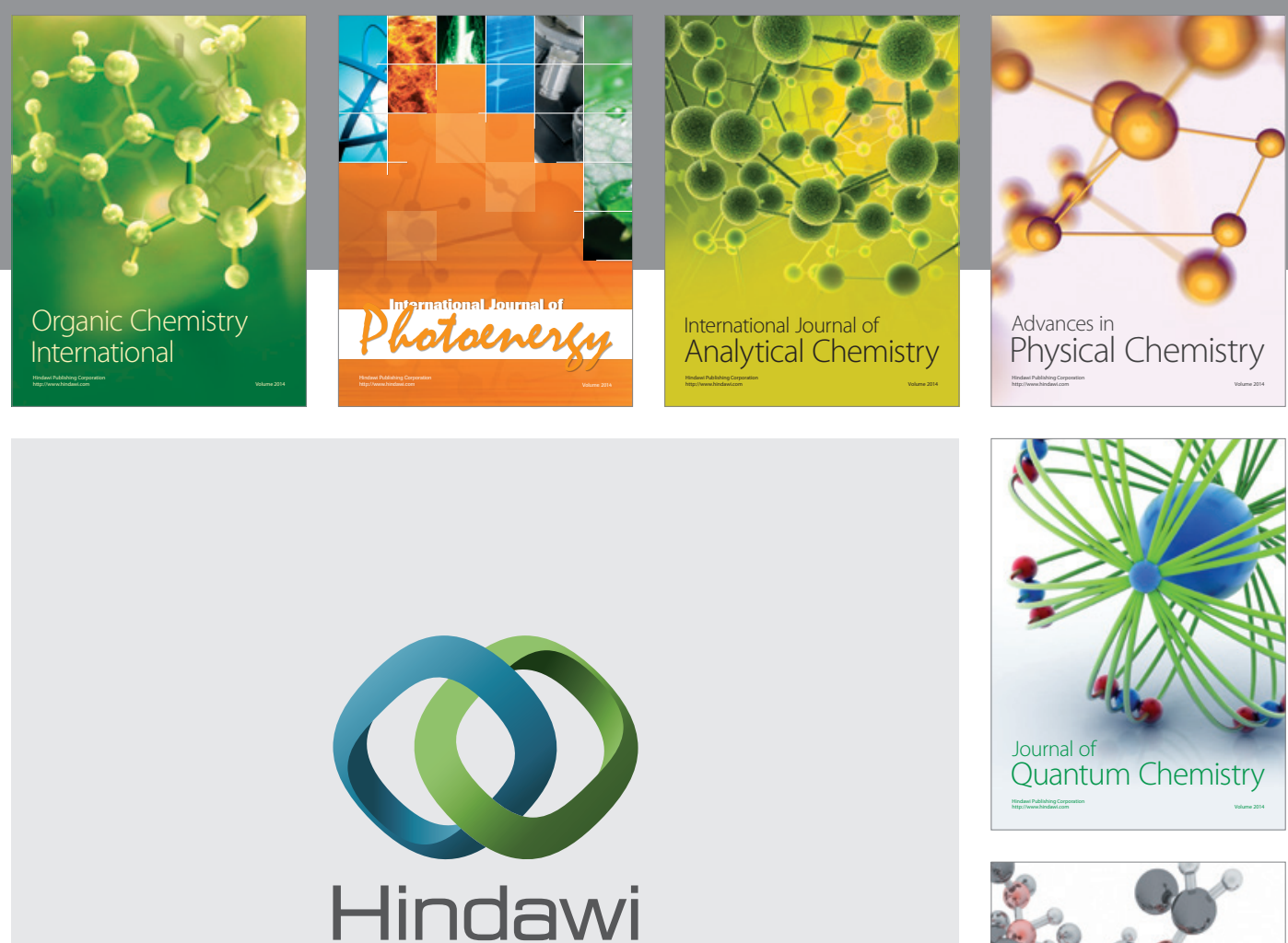

Submit your manuscripts at

http://www.hindawi.com

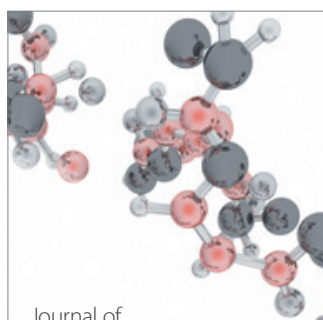

Analytical Methods

in Chemistry

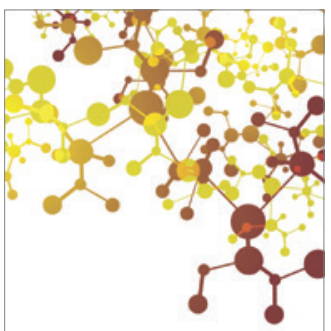

Journal of

Applied Chemistry

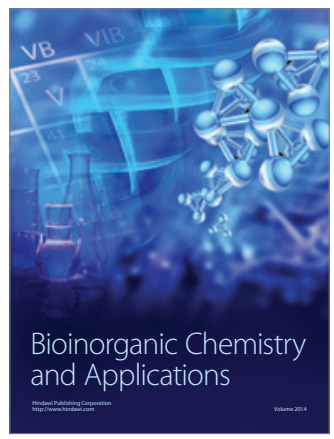

Inorganic Chemistry
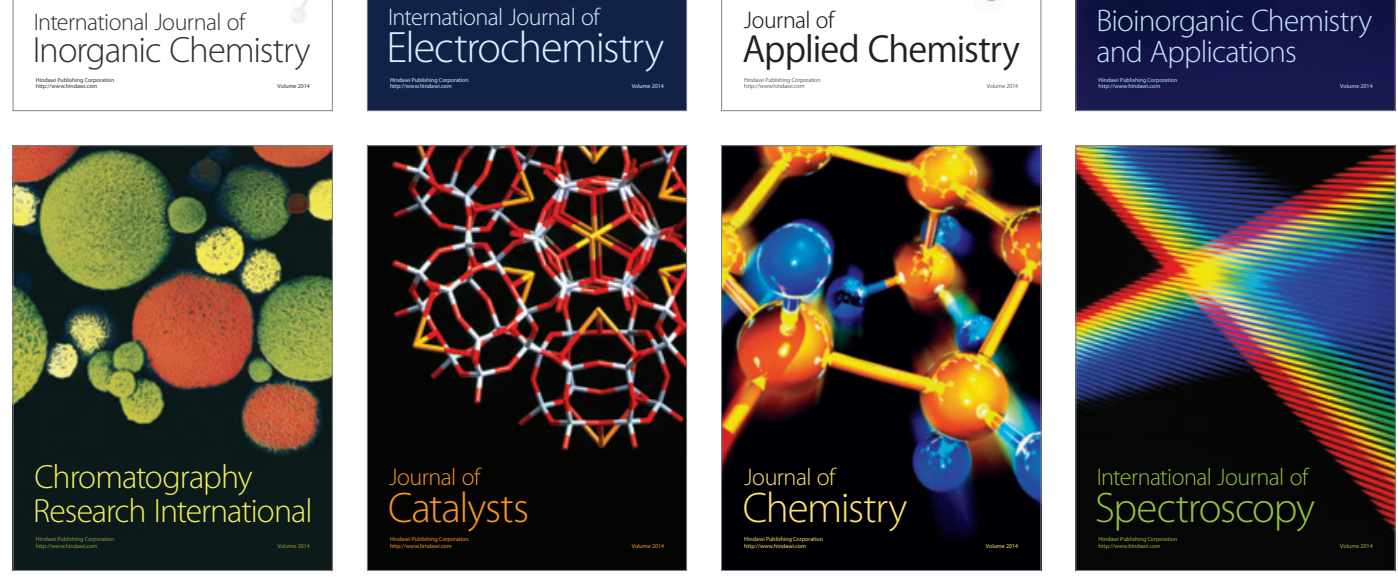